\title{
lodine-125 seed implantation for residual hepatocellular carcinoma or cholangiocellular carcinoma in challenging locations after transcatheter arterial chemoembolization: Initial experience and findings
}

\author{
Jie Li, MD*, Lijuan Zhang, MD²*, Zonggiong Sun, MD*, Yuxi Ge, MD*, Han Xiao, MD*, Qigen Xie, MD', \\ Shudong Hu, MD ${ }^{3 *}$ \\ 'Department of Interventional Radiology, the Affiliated Hospital of Jiangnan University (Wuxi $4^{\text {th }}$ People's Hospital), Wuxi, Jiangsu Province, \\ China, ${ }^{2}$ Department of Radiology, Wuxi People's Hospital of Nanjing Medical University. Wuxi, Jiangsu, China, ${ }^{3}$ Department of Radiology, \\ the Affiliated Hospital of Jiangnan University (Wuxi $4^{\text {th }}$ People's Hospital), Wuxi, Jiangsu Province, China, ${ }^{4}$ Department of Traditional Chinese \\ Medicine, the Affiliated Hospital of Jiangnan University (Wuxi $4^{\text {th }}$ People's Hospital). Wuxi, Jiangsu Province, China \\ *Jie Li, Lijuan Zhang, Zongaiong Sun, Ge Yuxi, Han Xiao, Shudong Hu contributed equally to this work.
}

\begin{abstract}
Purpose: To evaluate the clinical efficacy and safety of computed tomography (CT)-guided iodine-125 ( $\left.{ }^{125} \mathrm{I}\right)$ seed implantation (ISI) for hepatocellular carcinoma (HCC) or cholangiocellular carcinoma (CCC) lesions in challenging locations after transcatheter arterial chemoembolization (TACE).

Material and methods: A retrospective single-center review of 24 patients with HCC or CCC tumors in challenging locations (hepatic dome or close to the heart/diaphragm/hepatic hilum) was conducted. Patients who underwent CT-guided ${ }^{125}$ I implantation from May 2014 to January 2019 were recruited. Patients' demographics and details including technical success, treatment response, patient survival, and complication rate were also evaluated.

Results: Treated tumors were located in the hepatic dome $(n=10 ; 41.7 \%)$, subcapsularly $(n=6 ; 25 \%)$, close to the heart $(n=3 ; 12.5 \%)$, and in the liver hilum $(n=5 ; 20.8 \%)$. The mean maximum diameter of tumors in challenging locations was $40.08 \pm 11.34 \mathrm{~mm}$ (range, $25-68 \mathrm{~mm})$. TACE $(2 \pm 1,1-4$ times) was applied before ISI. There were 27 ISI treatments administered ( 3 patients also received supplemental ISI). The total number of implanted seeds was 1,160, with mean $48 \pm 16$ seed per patient (range, 30-90 seeds). The mean $\mathrm{D}_{90}$ value for ISI was $125 \mathrm{~Gy}$. Technical success rate was $100 \%$, while a complete response + partial response (CR + PR) was documented in $70.83 \%, 79.17 \%$, $83.33 \%$, and $79.17 \%$ of patients at $3,6,12$, and 24 months post-ISI, respectively. There were no major complications, although 2 cases experienced ${ }^{125} \mathrm{I}$ seed transfer to the diaphragm, and 1 case experienced transfer to the heart cavity.

Conclusions: CT-guided ISI for HCC or CCC lesions in challenging locations after TACE is both highly effective and safe.

J Contemp Brachytherapy 2020; 12, 3: 233-240 DOI: https://doi.org/10.5114/jcb.2020.96863
\end{abstract}

Key words: liver neoplasms, iodine-125, computed tomography, neoplasm, residual, chemoembolization, therapeutic.

\section{Purpose}

In men, hepatocellular carcinoma (HCC) is the second leading cause of cancer deaths worldwide. It has been estimated that China accounted for approximately $50 \%$ of the number of new HCC cases and deaths that occurred worldwide in 2012 [1,2].
Hepatocellular carcinoma or cholangiocellular carcinoma (CCC) are known to result in tumors in challenging locations, which have been classified as difficult lesions for laparoscopic liver resection [3]. International surveys have indicated that laparoscopic liver resection is performed less frequently for postero-superior segments than for other favorable segments (segments 2-6) [4]. 
As a standard treatment for stage B HCC, transcatheter arterial chemoembolization (TACE) is recommended according to the Barcelona clinic liver cancer (BCLC) staging system $[5,6]$. However, some scholars have performed pre-resection HCC embolization and observed that the proportion of HCC embolism achieving complete necrosis after surgery was only 13\% [7]. A follow-up review after one or several sessions of TACE showed that in most cases of HCC, loss of iodized oil occurs after embolization. Furthermore, this continues to occur after repeated sessions of TACE. Consequently, TACE of HCC needs to be repeated or combined with other treatments as early as possible after initial treatment. For patients with unresectable advanced HCC, treatment combining sorafenib can prolong patient's overall survival [8].

Iodine-125 $\left({ }^{125} \mathrm{I}\right)$ seed implantation (ISI) is being increasingly used as a minimally invasive treatment and in the treatment of intrahepatic and extrahepatic tumors, as it has been shown to achieve a better effect $[9,10]$. Combined TACE treatment for HCC can result in prolonged survival [11,12]. To our knowledge, the ISI after TACE treatment of local residual HCC or CCC tumors in challenging locations has limited coverage

Table 1. Baseline characteristics of the patients $(n=24)$

\begin{tabular}{lc} 
Variables & Values \\
\hline Age (years) & $58.35 \pm 5.602$ \\
\hline Gender (male/female) & $18 / 6$ \\
\hline HBsAg (positive/negative) & $19 / 5$ \\
\hline ECOG PS & 20 \\
\hline 0 & 4 \\
\hline 1 & \\
\hline Child-Pugh class & 19 \\
\hline A & 5 \\
\hline B & 2 \\
\hline Macrovascular invasion & 22 \\
\hline Yes & 18 \\
\hline No & 18 \\
\hline Extrahepatic metastasis & 7 \\
\hline Yes & 17 \\
\hline No & \\
\hline Tumor number & \\
\hline Solitary & \\
\hline Multiple & \\
\hline Liver cirrhosis & \\
\hline Yes & \\
\hline No & \\
\hline
\end{tabular}

Continuous variables were expressed as mean \pm standard deviation, HBSAghepatitis B surface antigen, ECOG PS - Eastern Cooperative Oncology Group performance status in the literature. Thus, we conducted this retrospective study to evaluate the safety and efficacy of ISI for the treatment of HCC or CCC lesions in challenging locations following TACE.

\section{Material and methods \\ Ethical approval of the study protocol}

This study was approved by the ethics committee of the Affiliated Hospital of Jiangnan University, China. The retrospective study collecting patients' information was approved by our institutional review board, which waived the requirement of informed patient consent. However, all patients were informed about the technique itself as well as possible benefits and complications and provided written informed consent prior to the procedure.

Because there is a lack of specific guidelines about challenging locations in HCC or CCC, our team applied a multidisciplinary experience of several departments [13]. For the treatment of lesions at these challenging locations, the decisions were made by senior doctors from the hepatobiliary surgery, interventional, and radiology departments. The doctors discussed the treatment options at different time points as well as the puncture path of ${ }^{125} \mathrm{I}$ seed implantation.

\section{Patients}

This retrospective study (between May 2014 and January 2019) was conducted in a single center, and enrolled 24 patients (18 males, 6 females; age range, 43-72 years, mean, $58.35 \pm 5.60$ years). Patients were diagnosed with HCC or CCC lesions in challenging locations and underwent TACE followed by ISI performed immediately after. After single or multiple TACE treatments, patients were observed as still having local residual tumor activity in locations considered to be special (these included hepatic dome and close to the heart/diaphragm/hepatic hilum at a distance $<1 \mathrm{~cm}$ ). Therefore, ISI was used to further control the local tumor. Patients with tumors not situated in the afore-mentioned anatomical locations were excluded from the study. None of the tumors in the group had invaded the adjacent diaphragm, and no tumors ruptured before ISI. All cases were unable to tolerate surgery due to poor cardiopulmonary function or they refused surgical resection for personal reasons. There were 2 patients who had tumors located close to the hepatic hilum, and portal vein branch invasion was noted. The diagnosis and treatment of HCC or CCC patients in this group was based on the 'standardization of diagnosis and treatment for hepatocellular carcinoma (2017 edition)' [14]. There were 19 confirmed cases with hepatitis B-associated liver cirrhosis, 1 case with hepatitis $C$, and 1 case with schistosomiasis-associated cirrhosis of the liver. Before ISI, there were 3 cases of bone metastasis, 2 cases of pulmonary metastasis, and 1 case with pulmonary metastasis and portal vein branch invasion. Each patient underwent laboratory tests (including routine blood tests, liver function, and coagulation tests) at least 24 hours prior ISI. Presurgical imaging consisted of contrast-enhanced computed tomography (CT) or/and multi-plane MRI. All patients 
were Child-Pugh class A or B and the Eastern Cooperative Oncology Group (ECOG) performance status (PS) $0-1$, with a platelet count greater than $80 \times 10^{9} / 1$. All baseline information is presented in Table 1.

\section{Transcatheter arterial chemoembolization}

In this group of patients, TACE treatment involved selection of superficial femoral artery using the Seldinger technique (manipulation of a $5 \mathrm{~F} \mathrm{R}-\mathrm{H}$ catheter to the right-hand side; Terumo, Tokyo, Japan) into the peritoneal dry artery, and the tumor blood supply into the arteries was confirmed by angiography. Using an angiography image as a reference, the microcatheter (Progreat; Terumo, Tokyo, Japan) was then guided to the tumor arterial blood supply. Through the microcatheter, conventional chemoembolization was conducted by injecting emulsified liquids of iodized oil (approximately $30 \mathrm{ml}$; Andre Guerbet, Aulnay-sous-Bois, France) and pirarubicin $\left(20 \mathrm{mg} / \mathrm{m}^{2}\right)$ into the supply artery until the flow was stopped or reflux was noted. Chemoembolization drug dosage depended on the tumor size and vascularity. If a hepatic arteriovenous fistula or hepatic artery portal vein fistula was observed, the appropriate size of gelatin sponge was selected according to the size of fistula for the embolization treatment of fistula. Liver-enhanced computed tomography (CT) review was typically performed within 6-8 weeks to determine the next treatment plan. For those patients with HCC tumors in challenging locations, we employed ISI to further strengthen the control of locally residual active lesions.

\section{Implantation of ${ }^{125} I$}

The patient $\mathrm{CT}$ images were transferred to a treatment-planning system (TPS) (BT-RSI; Yuan Bo, Beijing, China). The target area was sketched and both the number of puncture needles (puncture tools provided by HTA, Beijing, China) and number of implanted ${ }^{125}$ I seeds (model-6711, length, $4.5 \mathrm{~mm}$, diameter, $0.8 \mathrm{~mm}$ ) were determined. The implantation in all patients of this group was completed using 16 rows of CT (Siemens 16-row CT; Siemens, Germany) and a planning target volume of $90 \%$ $\left(D_{90}\right)$ was estimated as $100-130$ Gy for ${ }^{125}$ I seeds, with 25.9 MBq activity.

Before fixing and positioning the line marker at the surface area of the lesion, patient was arranged on the CT examination bed with the most appropriate position selected. The routine CT scan was initiated, and the target area of the lesion was circled. By combining the line marker and CT scan position, we determined the body surface puncture area and using the TPS plan, we were able to determine the angle and depth of the puncture needle. Then, the number of puncture needles and seeds required for the procedure were calculated. For those with HCC tumors in challenging locations, we were obliged to choose the right angle of the approach to the puncture. This would permit the location of the hepatic dome, while avoiding passing through the diaphragm. The experience of our team suggested performing a limited number of punctures passes through the diaphragm and lungs without significant complications.
Local infiltration anesthesia was carried out using $2 \%$ lidocaine, and the patient was instructed to breathe in a relaxed manner. $T$ puncture needle was inserted in place after CT scan to confirm its location, the gun was implanted, and regressive needles injected individual ${ }^{125}$ I seeds with spacing $<1 \mathrm{~cm}$ between the seeds. Immediately after TPS verification, we performed another CT scan after seed implantation, and assessed the matched peripheral dose. If a cold spot was noted in the local area, we replanted the seeds. The spacing arrangement of seeds followed the principle of gradual thinning outward from the center of the lesion. Further details on implantation of ${ }^{125}$ I seeds are described in previous studies by our team $[10,15,16]$.

\section{Follow-up}

All patients in the group were regularly reviewed (outpatient or inpatient). After the implantation, contrast-enhanced CT was performed 6-8 weeks after TACE or ISI, and if the lesion was stable, the review time was extended. Local control rate was evaluated according to the modified response evaluation criteria in solid tumors (MRESIST) [17]. On this basis, we assessed local control of HCC tumors in challenging locations after $3,6,12$, and 24 months after ISI (Table 2). HCC follow-up review time was in accordance with the guidelines. We routinely performed blood tests, liver function, alpha-fetoprotein (AFP), carbohydrate antigen (CA)19-9, and CA125 on every patient admission. Overall survival was defined as the interval between the first seed implant and either death or last follow-up. If lesions had progressed or there was distant transfer, we continued with TACE, TACE and ISI, or combined TACE with other therapies.

All patients were monitored for 4 hours after ISI and were discharged on the next day. Technical success, treatment response, patient survival, and complication rates were evaluated. The follow-up protocol consisted of clinical visits and contrast-enhanced CT at 1, 3, 6, 12, and 24 months. Complications were classified as major or minor according to international reporting standards [18]. Pain scores were recorded using a 0 (no pain) to 10 (maximum pain) visual-analogue score at 2 hours after the procedure and on 1-day post-ISI.

Table 2. Local control of $24 \mathrm{HCC}$ or CCC tumors in challenging locations after ${ }^{125}$ I seed implantation following TACE treatment

\begin{tabular}{lccccc}
$\begin{array}{l}\text { Follow-up time } \\
\text { (months) }\end{array}$ & \multicolumn{3}{l}{ Local control efficacy } & \multicolumn{2}{c}{$\begin{array}{c}\text { Local control rate } \\
\text { (CR + PR/total) }\end{array}$} \\
\cline { 2 - 5 } & CR & PR & SD & PD & \\
\hline 3 & 11 & 6 & 4 & 3 & $70.83 \%$ \\
\hline 6 & 13 & 6 & 5 & 0 & $79.17 \%$ \\
\hline 12 & 13 & 7 & 4 & 0 & $83.33 \%$ \\
\hline 24 & 12 & 7 & 3 & 2 & $79.17 \%$
\end{tabular}

$C R$ - complete response, $P R$ - partial response, $S D$ - stable disease, $P D$ - progressive disease 


\section{Statistical analysis}

The survival curves and statistical analyses were performed using GraphPad Prism, version 5 (Avenida, CA, USA). Continuous variables were expressed as mean \pm standard deviation. Overall survival was displayed using survival curves. Follow-up time started from the date of seed implantation.

\section{Results}

\section{Patients and treatment-related conditions}

A total of 24 HCC or CCC tumors in challenging locations in 24 patients were treated with CT-guided ISI implantation after TACE. TACE $(2 \pm 1,1-4$ times $)$ was employed before ISI. Enrolled patients with tumors were located in the hepatic dome $(n=10 ; 41.7 \%)$, subcapsularly $(n=6 ; 25 \%)$, close to the heart $(n=3 ; 12.5 \%$, all located less than $1.5 \mathrm{~cm}$ from the heart), and the liver hilum $(n=5 ; 20.8 \%)$ (Figures 1 and 2$)$. The maximum diameter average of the aforementioned tumors was $40.08 \pm 11.34 \mathrm{~mm}$ (range, $25-68 \mathrm{~mm}$ ). Tumor location and size are reported in Table 3. Alpha-fetoprotein was $\geq 400$ in $7 / 24$ patients. The pathological status for 11 patients was hepatocellular carcinoma, intrahepatic cholangiocarcinoma for 9 patients, and the remaining 4 was mixed. Six cases had tumor staging of III b by ex-
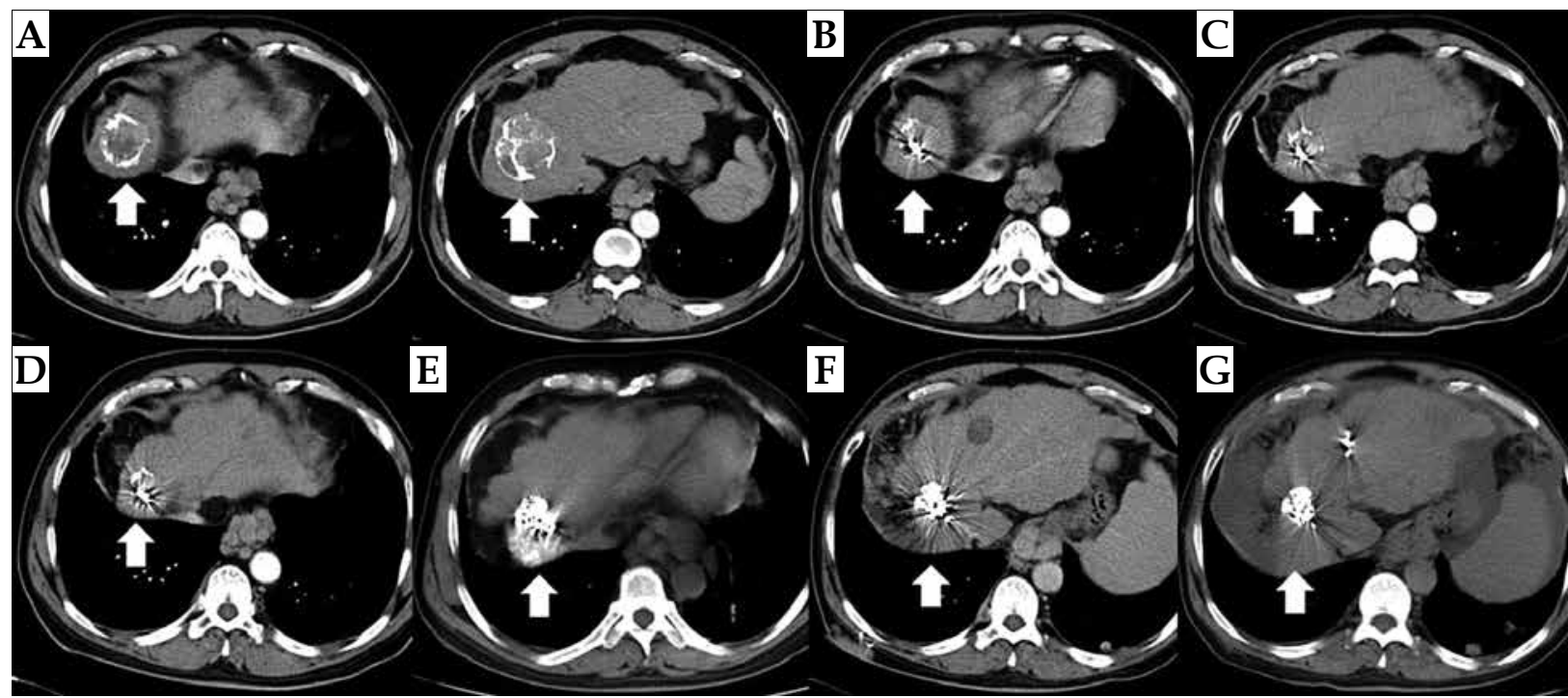

$\mathrm{G}$

Fig. 1. After TACE treatment of HCC tumors in challenging locations, we located tumors in the hepatic dome after 4 months. We were unable to achieve complete embolism or completely inactivate the lesion. However, 2 months after repeated TACE and ISI, we attained good control of locally active residual lesions. A) After repeated TACE of HCC tumors in challenging locations, we were unable to achieve complete embolism and locally residual active tumors were still present; B) Findings at 3 months after repeated TACE followed by ISI; C, D) Review of PR at 3 months and 7 months after ISI, respectively; E) At 12 months after ISI, TACE was repeated and complete embolism was achieved; F, G) Review of CR at 18 months and 22 months after ISI, respectively, and examination of new intrahepatic metastasis lesions after ISI treatment
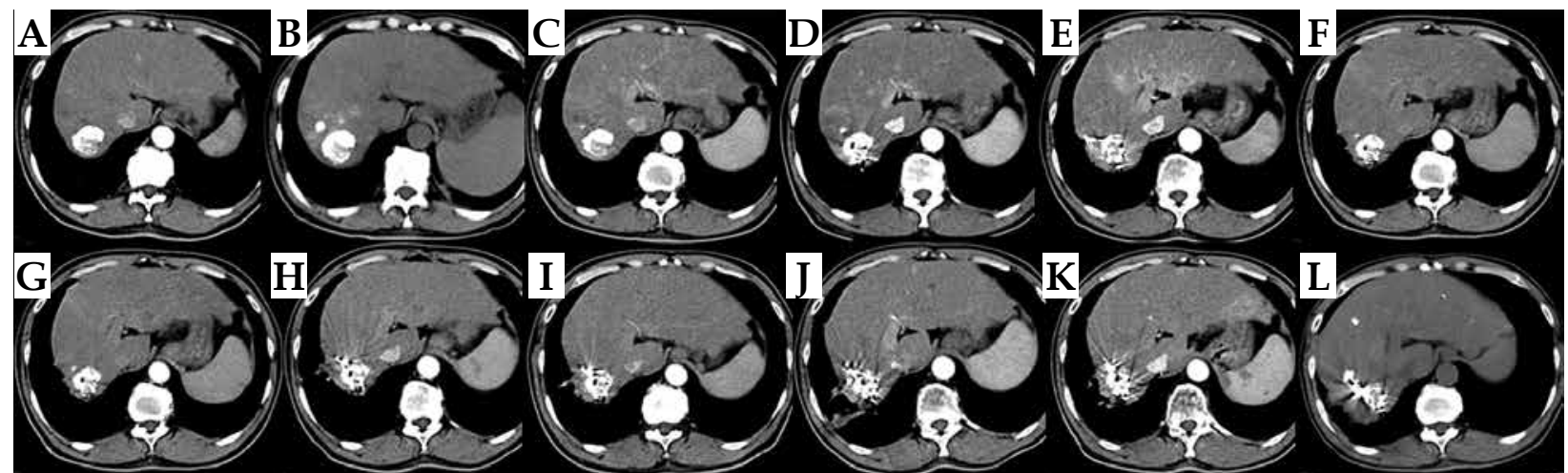

Fig. 2. After three TACE treatments for HCC tumors located in the subcapsular area, residual tumor activity persisted. However, the timing of ISI treatment resulted in well-controlled tumors. A-C) After the third round of TACE, patients with HCC still had active disease in tumors in challenging locations; D, E) Findings after two consecutive rounds of ISI in 4 months; F, G) Review of PR at 8 months and 12 months after ISI, respectively; H) Repeated ISI for local, residual disease; I-K) Review of CR at 18 months, 21 months, and 25 months after ISI, and mild radioactive pneumonia; L) Review at 28 months, after TACE was performed before $\mathrm{CR}$ was achieved. The Figure shows iodized oil deposition in new intrahepatic lesions 
Table 3. HCC or CCC tumors in challenging locations and treatment-related conditions

\begin{tabular}{|c|c|c|c|c|c|c|c|c|}
\hline $\begin{array}{l}\text { Case } \\
\text { no. }\end{array}$ & $\begin{array}{c}\text { Tumor } \\
\text { size }(\mathrm{mm}) \\
\end{array}$ & Location & $\begin{array}{c}\text { AFP } \\
(\mathrm{ng} / \mathrm{ml}) \\
\end{array}$ & $\begin{array}{l}\text { CA125 } \\
(\mathrm{U} / \mathrm{ml}) \\
\end{array}$ & $\begin{array}{l}\text { CA19-9 } \\
(\mathrm{U} / \mathrm{ml}) \\
\end{array}$ & Staging & $\begin{array}{c}\text { Number of } \\
\text { TACE sessions }\end{array}$ & $\begin{array}{c}\text { Number of } \\
\text { seed implants }\end{array}$ \\
\hline 1 & 49 & Hepatic dome & 178.4 & 235.7 & 89.55 & Ila & 2 & 60 \\
\hline 2 & 34 & Close to the liver hilum & $\geq 1210$ & / & / & IIIb & 1 & 40 \\
\hline 3 & 33 & Subcapsular & / & 44.7 & 35.8 & Ila & 1 & 40 \\
\hline 4 & 68 & Close to the heart & 43.2 & 65.2 & 203.2 & IIIb & 2 & 90 \\
\hline 5 & 34 & Hepatic dome & / & 56.7 & 87.45 & Ila & 1 & 40 \\
\hline 6 & 49 & Hepatic dome & $\geq 1210$ & 35.5 & / & Ila & 1 & 50 \\
\hline 7 & 34 & Subcapsular & / & 88.9 & 97.21 & Ila & 1 & 60 \\
\hline 8 & 25 & Close to the liver hilum & / & 37.43 & 56.32 & IIIa & 2 & 40 \\
\hline 9 & 38 & Close to the heart & / & 45.3 & 78.7 & Ila & 3 & 40 \\
\hline 10 & 28 & Subcapsular & $\geq 1210$ & 21.2 & 10.05 & la & 1 & 30 \\
\hline 11 & 55 & Hepatic dome & $\geq 1210$ & / & l & IIIb & 3 & 70 \\
\hline 12 & 36 & Hepatic dome & 130.5 & 76.44 & 549.1 & Ila & 3 & 40 \\
\hline 13 & 26 & Hepatic dome & 43.3 & l & l & $\mathrm{Ib}$ & 2 & 30 \\
\hline 14 & 58 & Close to the heart & $\geq 1210$ & / & l & la & 2 & 70 \\
\hline 15 & 50 & Close to the liver hilum & / & 64.82 & 39.16 & IIIb & 4 & 70 \\
\hline 16 & 38 & Subcapsular & 67.8 & 79.3 & 55.3 & $\mathrm{Ilb}$ & 2 & 40 \\
\hline 17 & 54 & Hepatic dome & $\geq 1210$ & 33.7 & l & $\mathrm{lb}$ & 2 & 70 \\
\hline 18 & 33 & Hepatic dome & / & l & l & $\mathrm{lb}$ & 1 & 50 \\
\hline 19 & 26 & Subcapsular & / & 41.3 & 75.22 & IIla & 1 & 30 \\
\hline 20 & 30 & Hepatic dome & 57.22 & / & 87.2 & $\mathrm{lb}$ & 2 & 30 \\
\hline 21 & 36 & Subcapsular & 35.3 & 54.1 & l & Ila & 2 & 40 \\
\hline 22 & 43 & Close to the liver hilum & / & 32.44 & 219.1 & IIIb & 2 & 40 \\
\hline 23 & 47 & Close to the liver hilum & $\geq 1210$ & 434.2 & 44.2 & IIIb & 2 & 50 \\
\hline 24 & 38 & Hepatic dome & 11.7 & / & 65.4 & Ila & 2 & 40 \\
\hline
\end{tabular}

AFP-alpha-fetoprotein, CA125 - carbohydrate antigen 125, CA19-9 - carbohydrate antigen 19-9, "/" meaning for the normal range.

Tumor size refers to the magnitude of HCC-LBD. The number of TACE episodes before ISI are shown. Staging is based on the clinical staging of China's 2017 version of liver cancer guidelines [15]

trahepatic metastasis (see Table 3 for more information on cancer and treatment). All patients in this group had good follow-up attendance (30.42 \pm 13.58 months, 10-56 months, and overall survival in follow-up) as shown in Figure 3.

\section{Efficacy of ISI after TACE}

An angulated indirect approach through the normal liver parenchyma was performed in HCC or CCC tumors located at hepatic dome, whilst an indirect approach through the normal liver parenchyma was performed in the remaining HCC or CCC tumors. Technical success rate was $100 \%$; twenty-four patients with HCC or CCC tumors in challenging locations were treated with $27 \mathrm{ep}$ isodes of ISI (3 of which underwent supplemental ${ }^{125} \mathrm{I}$ seed implantation). The total number of implanted seeds

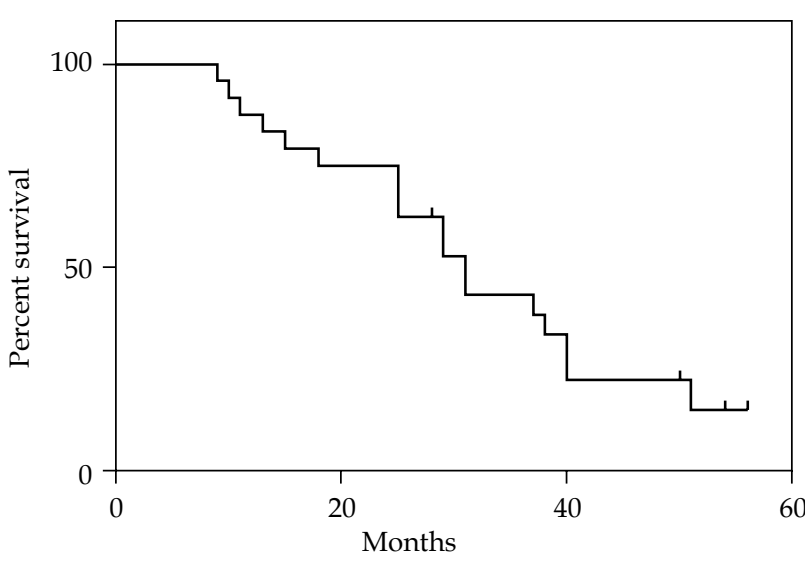

Fig. 3. Overall follow-up survival curve 
was 1,160 , with a mean seed count of $48 \pm 16$ per patient (range, 30-90 seeds). The mean $\mathrm{D}_{90}$ value for implanted ${ }^{125}$ I seeds was $125 \mathrm{~Gy}$.

CT examination was performed 6-8 weeks after TACE/ISI, and tumor control was assessed based on the measurement of diameter of the tumor during CT enhancement. CT review based on changes in tumor size are shown in Figures 1 and 2. Complete response + partial response (CR + PR) was documented in $70.83 \%$, $79.17 \%, 83.33 \%$, and $79.17 \%$ of patients at $3,6,12$, and 24 months after ISI, respectively. At the early stages of follow-up, there were 3 cases of progressive disease (PD) associated with HCC tumors in challenging locations due to irregular tumor shape. The number and arrangement of ISI as well as local control was good after the repeated implantation of ${ }^{125}$ I seeds. At 24 months of follow-up, 2 patients were found to have a new HCC tumor in a challenging location. Patients with multiple metastases at the end of 24-month of follow-up refused to continue with ISI treatment.

\section{Adverse events}

All ISI procedures were completed successfully. No major complications including serious diaphragmatic injuries (e.g., diaphragmatic disruption, paralyses, increase in diaphragm thickness, or right pleural effusions) were observed. There was no procedure-related mortality. During surgery, 3 cases were implanted through the diaphragm and 1 patient exhibited a small degree of pneumothorax. The patient had less than $20 \%$ of blood appearance in a phlegm, no detailed chest tightness, shortness of breath, and was discharged after two days of observation. During follow-up, 2 patients were noted with ${ }^{125} \mathrm{I}$ seeds transferred to the diaphragm, and 1 patient experienced seed transfer to the heart cavity. However, close follow-up was performed in all patients and they were reported to be well. The mean pain score according to the visual analogue scale (VAS) was $1.31 \pm 1.23$ during ISI, $0.82 \pm 0.55$ at two hours after ISI, and $0.02 \pm 0.21$ at day 1 post-ISI. After surgery, the blood, liver, and kidney functions were normal. One individual experienced subcutaneous implant metastasis.

\section{Discussion}

Treatment of HCC or CCC tumors with challenging locations is associated with technical difficulties, which means that surgery, radiotherapy, and microwave treatment cannot be used. Laparoscopic liver resection (LLR) is now widely performed, but it is difficult to accomplish in some anatomical locations, such as the postero-superior segments (S7 and S8) and caudate lobe (S1) [19]. Ablation therapy has also been used frequently in the selection and guidance of surgical entry routes [20]. TACE is a classic treatment for HCC and is performed through the hepatic artery approach to find supply vessels of HCC. It permits further ultra-selective treatment and is not affected by challenging locations, which are evident in such tumors $[14,21]$. However, even after TACE and repeated multiple TACE treatments, a large proportion of patients with HCC still cannot attain complete cure by embolism $[7,22]$. Therefore, other treatments must be combined as early as possible after treatment failure.

ISI implantation has been gradually developed in clinical practice as a new, minimally invasive treatment for unresectable HCC therapy. Previous studies have shown that TACE combined with ISI permit to control liver tumors by local therapy, which is beneficial for patients [11,12]. Basic experimental studies have confirmed that ${ }^{125}$ I seed implantation control tumors mainly through apoptosis and G2/M cell cycle arrest [23,24]. According to the results of this and our former study [25], ISI can be used safely and performed in challenging hepatic locations, including the hepatic dome, subcapsular location, or locations proximal to the heart and diaphragm. Additionally, ISI in these locations was reported to achieve very satisfactory local control rates $(20 / 24$ tumors in 12 months, $83.33 \%$ ). However, it must be noted that each hepatic location warrants a different approach and an individualized strategy in order to achieve total tumor control without significant complications. In the present study, ISI was performed by experienced physicians under CT guidance, sometimes at a distance of $1 \mathrm{~cm}$ from the diaphragm. Importantly, ISI treatment did not result in artificial diaphragm perforation, rupture, thoracic ascites, or other conditions. Moreover, Chinese scholars have conducted a lot of research on HCC using portal vein tumor thrombus combined with seed implantation [26].

Ablation therapy is recommended for patients with small HCC ( $\leq 3 \mathrm{~cm}$ in number and diameter) [27]. However, the use of ablation in the liver beneath the diaphragm is often a limiting cause of pneumothorax [28]. Pain during a procedure in challenging hepatic tumor locations is quite common after ablation. Hakime et al. clarified that artificial ascites prevent only immediate post-procedural pain, which re-appears intensively after 4 days [29]. ISI is associated with a slight pain, which is an advantage when comparing ablation treatments. In a study by Fang, combined ${ }^{125}$ I seed implantation based on TACE and radiofrequency ablation (RFA) in the treatment of subcapsular HCC was used and demonstrated to improve efficacy [30].

CT-guided high-dose-rate brachytherapy (CTHDRBT) is an effective therapy for attaining good local tumor control (LTC) in both primary liver tumors, e.g., HCC and CCC as well as metastases [31], especially when the tumor diameter is larger than $5 \mathrm{~cm}$ [32]. One study found that $8.5 \%$ of tumors (from a total of 212) showed local progression after a mean LTC of 21.1 months [33]. CT-HDRBT is a promising therapy for unresectable HCCs that are not suitable for thermal ablation, because their mean size exceeds $7.1 \mathrm{~cm}$; the mean follow-up period reported was 12.8 months, with a local progression rate of $6.7 \%$ [34]. Treatment of patients with large-sized HCC remains challenging, and survival of patients in advanced tumor stages is limited. Some studies have reported that treatment with TACE and subsequent CTHDRBT results are associated with a very promising survival rate for patients with unresectable HCC [35]. From the economic perspective, CT-HDRBT is less expensive 
than RFA and MWA [36]. Therefore, the combination of CT-HDRBT with TACE offers a dual advantage in the treatment of tumors. In this study, ISI was only used as a local complementary treatment for residual HCC or CCC after TACE.

In this study, the local control rate of HCC or CCC tumors in challenging locations was quite poor. Most probably, this was caused by too sparse seeds' arrangement, resulting in poor local control rate of lesions, which continued after two further replanting sessions of ${ }^{125}$ I seed. We believe this could be due to more advanced disease in the subsequent follow-up of local control rate. Some lesions are difficult to treat due to their irregular morphology. Consequently, in order to achieve good local control effect, the seed dosage may be rather biased. For 2 patients, it was not possible to continue local seed implantation to strengthen the control of local lesions. In a small cohort of 24 patients, this may adversely influence the efficacy of the technique. ISI treatment of patients with HCC or CCC tumors in challenging locations is not strict due to the patient's cardiopulmonary function requirements. However, it can be repeated for ISI treatment at any time as long as the seed covered lesions are estimated to exert local control. Furthermore, patients appear to easily accept ISI treatment, they recover fast, and the application of the technique is relatively easy.

In this group, only 1 patient showed subcutaneous planting metastasis. Analysis of the approach to the angle of choice for the patient can be technically quite difficult. We recommend that to minimize the number of punctures, in vitro optimization should be taken as a reference before needle selection. Unlike ablation therapy, ISI can melt the puncture path at the same time, which is the main drawback of ISI.

TACE combined with ISI to treat patients with HCC or CCC tumors in challenging locations is very safe and efficient. Patients generally experience little pain and they recover quickly. Although our study provides positive findings, some limitations should be noted. Our study was of a retrospective nature and the small patient sample size may not be generalizable, especially for tumors in certain challenging locations, such as close to the heart or to the liver hilum. Consequently, our findings are relatively limited and larger studies are required to confirm our observations.

\section{Conclusions}

CT-guided ${ }^{125} \mathrm{I}$ seed implantation for the treatment of challenging HCC or CCC tumors (diameter range, $25-68 \mathrm{~mm}$ ), including those located in the hepatic dome or close to heart/diaphragm/hepatic hilum, resulted in satisfactory efficacy and safety rates. These experimental approaches should be further investigated to validate our findings and to provide encouraging data.

\section{Compliance with ethical standards}

All procedures performed were in accordance with the 1964 Helsinki Declaration and its later amendments or comparable ethical standards, and ethical approval was obtained from the Affiliated Hospital of Jiangnan University.

Informed consent was obtained from all participants included in the study.

\section{Disclosure}

This work was supported in a part by grants from the Jiangsu Provincial Commission of Health and Family Planning (Q201615 to Jie Li), the Scientific Research Project of Wuxi Health and Family Planning Commission (Q201920 to Jie Li, MS201643 to Zongqiong Sun), and Wuxi Young Medical Talents Project (QNRC060 to Han Xiao)

The authors report no conflict of interest.

\section{References}

1. Torre LA, Bray F, Siegel RL et al. Global cancer statistics, 2012. CA Cancer J Clin 2015; 65: 87-108.

2. Chen W, Zheng R, Baade PD et al. Cancer statistics in China, 2015. CA Cancer J Clin 2016; 66: 115-132.

3. Araki K, Kubo N, Watanabe A et al. Systematic review of the feasibility and future of laparoscopic liver resection for difficult lesions. Surg Today 2018; 48: 659-666.

4. Hibi T, Cherqui D, Geller DA et al. Expanding indications and regional diversity in laparoscopic liver resection unveiled by the International Survey on Technical Aspects of Laparoscopic Liver Resection (INSTALL) study. Surg Endosc 2016; 30: 2975-2983.

5. Bruix J, Reig M, Sherman M. Evidence-based diagnosis, staging, and treatment of patients with hepatocellular carcinoma. Gastroenterology 2016; 150: 835-853.

6. Gnutzmann D, Kortes N, Sumkauskaite M et al. Transvascular therapy of hepatocellular carcinoma (HCC), status and developments. Minim Invasive Ther Allied Technol 2018; 27: 69-80.

7. Liu Y, Yang R. Preoperative combined with postoperative chemoembolization can improve survival in patients with hepatocellular carcinoma: a single-center study. J Vasc Interv Radiol 2009; 20: 472-483.

8. Ren B, Wang W, Shen J et al. Transarterial chemoembolization (TACE) combined with sorafenib versus TACE alone for unresectable hepatocellular carcinoma: a propensity score matching study. J Cancer 2019; 10: 1189-1196.

9. He C, Liu Y, Li Y et al. Efficacy and safety of computed tomography-guided 125I brachytherapy for lymph node metastatic from hepatocellular carcinoma. J Cancer Res Ther 2018; 14: 754-759.

10. Li J, Xie Q, Wang W et al. CT-guided implantation of $125 \mathrm{I}$ seed (permanent brachytherapy) for metastatic tumors of the hepatic portal system: Effectiveness and safety in 13 patients. Brachytherapy 2016; 15: 224-230.

11. Zhu ZX, Wang XX, Yuan KF et al. Transarterial chemoembolization plus iodine-125 implantation for hepatocellular carcinoma: a systematic review and meta-analysis. HPB (Oxford) 2018; 20: 795-802.

12. Li M, He J, Pan M et al. Iodine-125 implantation plus transarterial chemoembolization for the treatment of hepatocellular carcinoma of 3-5 cm: A propensity score matching study. Dig Liver Dis 2016; 48: 1082-1087.

13. Kovács G, Tagliaferri L, Valentini V. Is an Interventional Oncology Center an advantage in the service of cancer patients or in the education? The Gemelli Hospital and INTERACTS experience. J Contemp Brachytherapy 2017; 9: 497-498.

14. Bureau of Medical Administration, National Health and Family Planning Commission of the PRC. Standardization of 
diagnosis and treatment for hepatocellular carcinoma (2017 edition). Chin J Dig Surg 2017; 16: 635-647.

15. Li J, Zhang L, Xu W et al. Computed tomography-guided implantation of ${ }^{125} \mathrm{I}$ seeds brachytherapy for recurrent multiple pulmonary oligometastases: initial experience and results. J Contemp Brachytherapy 2017; 9: 132-138.

16. Li J, Zhang L, Xie Q et al. Comparison of clinical efficacy and complications of ${ }^{125} \mathrm{I}$ seed brachytherapy and stereotactic body radiation therapy for recurrent pulmonary metastases from colorectal carcinoma. J Contemp Brachytherapy 2018; 10: 360-367.

17. Lencioni R, Llovet JM. Modified RECIST (mRECIST) assessment for hepatocellular carcinoma. Semin Liver Dis 2010; 30: $52-60$.

18. Leoni CJ, Potter JE, Rosen MP et al. Classifying complications of interventional procedures: a survey of practicing radiologists. J Vasc Interv Radiol 2001; 12: 55-59.

19. Araki K, Kubo N, Watanabe A et al. Systematic review of the feasibility and future of laparoscopic liver resection for difficult lesions. Surg Today 2018; 48: 659-666.

20. Zhang D, Liang P, Yu X et al. The value of artificial pleural effusion for percutaneous microwave ablation of liver tumour in the hepatic dome: a retrospective case-control study. Int J Hyperthermia 2013; 29: 663-670.

21. European Association For The Study Of The Liver, European Organisation For Research And Treatment Of Cancer. EASLEORTC clinical practice guidelines: management of hepatocellular carcinoma. J Hepatol 2012; 56: 908-943.

22. Ikeda K, Kumada H, Saitoh $S$ et al. Effect of repeated transcatheter arterial embolization on the survival time in patients with hepatocellular carcinoma. An analysis by the Cox proportional hazard model. Cancer 1991; 68: 2150-2154.

23. Wang ZM, Lu J, Zhang LY et al. Biological effects of lowdose-rate irradiation of pancreatic carcinoma cells in vitro using 125I seeds. World J Gastroenterol 2015; 21: 2336-2342.

24. Dawson LA, Ten Haken RK, Lawrence TS. Partial irradiation of the liver. Semin Radiat Oncol 2001; 11: 240-246.

25. Li J, Zhang L, Xie Q et al. ${ }^{125}$ I seeds implantation for treating residual hepatocellular carcinoma located beneath the diaphragm after transcatheter arterial chemoembolization. Brachytherapy 2019; 18: 420-425.

26. Luo J, Zheng JP, Shao GL et al. Comparison of therapeutics effects of transcatheter arterial chemoembolization combined with iodine- 125 seed implantation and sorafenib for the treatment of hepatocellular carcinoma with portal vein tumor thrombosis. Zhonghua Gan Zang Bing Za Zhi 2018; 26 : 298-304.

27. Omata M, Cheng AL, Kokudo N et al. Asia-Pacific clinical practice guidelines on the management of hepatocellular carcinoma: a 2017 update. Hepatol Int 2017; 11: 317-370.

28. Miura H, Yamagami T, Terayama K et al. Pneumothorax induced by radiofrequency ablation for hepatocellular carcinoma beneath the diaphragm under real-time computed tomography-fluoroscopic guidance. Acta Radiol 2010; 51: 613-618.

29. Hakimé A, Tselikas L, Otmezguine $Y$ et al. Artificial ascites for pain relief during microwave ablation of subcapsular liver tumors. Cardiovasc Intervent Radiol 2015; 38: 1557-1562.

30. Fang SJ, Gao RJ, Zheng LY et al. Clinical value of (125) I radioactive seed implantation plus transcatheter arterial chemoembolization combined with radiofrequency ablation in treating patient with sub-capsular hepatocellular carcinoma. Zhonghua Yi Xue Za Zhi 2018; 98: 3853-3857.

31. F, Gebauer B. CT-guided high dose rate brachytherapy ablation of liver metastases. Imaging Med 2013; 5: 383-388.

32. Bretschneider T, Ricke J, Gebauer B et al. Image-guided highdose-rate brachytherapy of malignancies in various inner organs - technique, indications, and perspectives. J Contemp Brachytherapy 2016; 8: 251-261.

33. Collettini F, Schreiber N, Schnapauff D et al. CT-guided highdose-rate brachytherapy of unresectable hepatocellular carcinoma. J Strahlenther Onkol 2015; 191: 405-412.

34. Collettini F, Schnapauff D, Poellinger A, et al. Hepatocellular carcinoma: computed-tomography-guided high-dose-rate brachytherapy (CT-HDRBT) ablation of large $(5-7 \mathrm{~cm})$ and very large $(>7 \mathrm{~cm})$ tumours. J Eur Radiol 2012; 22: 1101-1109.

35. Schnapauff D, Tegel BR, Powerski MJ et al. Interstitial brachytherapy in combination with previous transarterial embolization in patients with unresectable hepatocellular carcinoma. J Anticancer Res 2019; 39: 1329-1336.

36. Kovács A, Iezzi R, Cellini F et al. Critical review of multidisciplinary non-surgical local interventional ablation techniques in primary or secondary liver malignancies. J Contemp Brachytherapy 2019; 11: 589-600. 